Katarzyna Buszko*1, Łukasz Pietrzykowski², Piotr Michalski², Agata Kosobucka², Wioleta Stolarek ${ }^{3}$, Tomasz Fabiszak ${ }^{4}$, Aldona Kubica ${ }^{2}$

${ }^{1}$ Department of Theoretical Foundations of Biomedical Science and Medical Informatics, Collegium Medicum, Nicolaus Copernicus University, Bydgoszcz, Poland

${ }^{2}$ Department of Health Promotion, Collegium Medicum, Nicolaus Copernicus University, Bydgoszcz, Poland

${ }^{3}$ Department of Pharmacology and Therapy, Ludwik Rydygier Collegium Medicum, Nicolaus Copernicus University, Bydgoszcz, Poland

${ }^{4}$ Department of Cardiology and Internal Diseases, Collegium Medicum, Nicolaus Copernicus University, Bydgoszcz, Poland

* Katarzyna Buszko and Łukasz Pietrzykowski have equally contributed to the present paper and should be considered to be the first authors

\title{
Validation of the Functioning in Chronic IIIness Scale (FCIS)
}

\author{
Corresponding author: \\ Łukasz Pietrzykowski \\ Department of Health Promotion, \\ Collegium Medicum, \\ Nicolaus Copernicus University \\ ul. Łukasiewicza $185-821$ Bydgoszcz, \\ Poland, tel.: 525855801 \\ e-mail: \\ lukasz.pietrzykowski@cm.umk.p
}

Medical Research Journal 2018; Volume 3, Number 2, 63-69 10.5603/MRJ.2018.001 Copyright (C) 2018 Via Medica ISSN 2451-2591

\begin{abstract}
Diagnosis of deficient areas in the functioning of patient with chronic disease is necessary to undertake the adequate therapeutic actions. The aim of the study was to validate a new self-reported questionnaire for patients with chronic disease assessing the impact of the disease on the patient, the patient's impact on the disease and the impact of the disease on patient's attitudes.

Results: The internal consistency of the questionnaire expressed by a-Cronbach coefficient $=0.855$, indicates its high reliability and homogeneity. The set of 24 items fulfilled the assumption of factor analysis: the determinant of correlation matrix was 0.001, Kaiser-Mayer-Olkin (K-M-O) statistic was 0.843 and the Bartlett' test of sphericity was statistically significant. The factor analysis was conducted using the principal component analysis with Varimax rotation. The scale and subscale levels were determined based on the percentiles scale.

Conclusion: The validation procedure revealed that FCIS is a reliable and homogeneous tool to measure patient's physical and mental functioning in the chronic illness. The set of items divided into 3 subscales allows evaluation of: the impact of the disease on the patient, the patient's impact on the disease and the impact of the disease on the patient's attitudes.
\end{abstract}

Key words: validation, chronic illness, scale

Med Res J 2018; 3 (2): 63-69

\section{Introduction}

Chronic disease affects all aspects of human life. The degree of interference is largely dependent on the severity of disease symptoms. The impact of the disease essentially covers all areas of human functioning, including: physical activity, emotional and spiritual sphere, and functioning in society [1-5]. The functioning limitation of patients with chronic disease results in lower self-value perception, deterioration in well-being, increase of anxiety and uncertainty about the future [6-10]. According to the available literature the influence of chronic disease on human life has been studied so far, assessing various but single aspects, eg quality of life, physical and mental functioning, level of disease acceptance, self-efficacy or health self-control location [11-13]. However, there are no tools to evaluate the overall of patient functioning. It seems reasonable to create a simple tool to assess the overall functioning of the patient in chronic disease. The tool should allow the identification of deficient areas in the functioning of patient with chronic disease in order to undertake the adequate therapeutic actions. The aim of the study was to validate a new self-reported questionnaire for patients with chronic disease assessing the impact of the disease on the patient, the patient's impact on the disease and the impact of the disease on patient's attitudes.

\section{Methods}

Study group

The study group consisted of 366 patients, 188 (51\%) females and 178 (49\%) males with coronary artery disease (CAD) treated with percutaneous coronary intervention (PCl). The study was conducted 
Table 1. Characteristics of patients

\begin{tabular}{lcc}
\hline Parametr & Category/value & $\begin{array}{c}\text { Number } \\
\text { of patients }\end{array}$ \\
\hline Age & $>65$ & $189(51 \%)$ \\
Education & Primary & $42(11 \%)$ \\
& Vocational & $123(34 \%)$ \\
& Secondary & $149(41 \%)$ \\
Place of residence & Higher & $52(14 \%)$ \\
& Town/Village & $104(28 \%)$ \\
Economic status & City & $262(72 \%)$ \\
& Bad & $18(5 \%)$ \\
& Acceptable & $73(20 \%)$ \\
& Good & $245(67 \%)$ \\
& Very good & $30(8 \%)$ \\
\hline
\end{tabular}

between March and July 2017 . The study population characteristics is displayed in Tab 1.

The questionnaire consists of 24 questions divided into three parts, with a catalogue of 5 answers added to each question. Each answer was graded from 1 to 5 points.

\section{The Functioning in Chronic Illness Scale (FCIS)}

Author: Aldona Kubica

Please, refer to each question by choosing (encircling) the answer which best reflects your opinion. There are no wrong answers - all answers are relevant as long as they are true.

\section{Part 1 - The impact of illness on the patient}

Patient's subjective assessment of the impact of illness on his / her life.

Maximal score - illness does not affect the patient's functioning

Minimal score - illness severely affects the patient's functioning

1. My physical capacity is similar as prior to the illness.

Definetely NOT (1); rather not (2); I do not know (3); rather yes (4); definitely YES (5)

2. The illness limits my physical activity.

Definetely NOT (1); rather not (2); I do not know (3); rather yes (4); definitely YES (5)

3. Despite the illness I consider myself a wholesome person.

Definetely NOT (1); rather not (2); I do not know (3); rather yes (4); definitely YES (5)

4. I have been a burden to my family ever since the illness began.

Definetely NOT (1); rather not (2); I do not know (3); rather yes (4); definitely YES (5)
5. Despite the illness I feel OK.

Definetely NOT (1); rather not (2); I do not know (3); rather yes (4); definitely YES (5)

6. I feel worse than I felt before the illness.

Definetely NOT (1); rather not (2); I do not know (3); rather yes (4); definitely YES (5)

7. My life is the same as prior to the illness.

Definetely NOT (1); rather not (2); I do not know (3); rather yes (4); definitely YES (5)

8. My illness limits my social and family contacts.

Definetely NOT (1); rather not (2); I do not know (3); rather yes (4); definitely YES (5)

Part 2 - The patient's impact on the illness.

Defining the patients' opinions on their impact on the course of illness.

Maximal score - the patient believes to have significant impact on the course of illness

Minimal score - the patient believes to have no impact on the course of illness

1. My illness exclusively results from my neglect.

Definetely NOT (1); rather not (2); I do not know (3); rather yes (4); definitely YES (5)

2. My illness is not my fault.

Definetely NOT (1); rather not (2); I do not know (3); rather yes (4); definitely YES (5)

3. I am primarily responsible for my future well- / ill-being.

Definetely NOT (1); rather not (2); I do not know (3); rather yes (4); definitely YES (5)

4. Only doctors are responsible for my future well- / ill-being.

Definetely NOT (1); rather not (2); I do not know (3); rather yes (4); definitely YES (5)

5. Regular medication intake may be beneficial my health.

Definetely NOT (1); rather not (2); I do not know (3); rather yes (4); definitely YES (5)

6. Medications are more likely to be harmful than beneficial.

Definetely NOT (1); rather not (2); I do not know (3); rather yes (4); definitely YES (5)

7. Regular daily physical activity may be beneficial my health.

Definetely NOT (1); rather not (2); I do not know (3); rather yes (4); definitely YES (5)

8. Due to my illness, I should limit my physical activity to minimum and avoid even lightest exercise.

Definetely NOT (1); rather not (2); I do not know (3); rather yes (4); definitely YES (5)

\section{Part 3 - The impact of illness on patient's attitude}

Defining the patient's attitude towards new circumstances of life. 
Maximal score - the patient holds a very optimistic view for the future

Minimal score - the patient holds a very pessimistic view for the future

1. My illness made me actively take care of my health. Definetely NOT (1); rather not (2); I do not know (3); rather yes (4); definitely YES (5)

2. I feel helpless against my illness.

Definetely NOT (1); rather not (2); I do not know (3); rather yes (4); definitely YES (5)

3. I believe that my health can improve.

Definetely NOT (1); rather not (2); I do not know (3); rather yes (4); definitely YES (5)

4. I suppose that my illness will cause a gradual decline of my well-being.

Definetely NOT (1); rather not (2); I do not know (3); rather yes (4); definitely YES (5)

5. I actively think about steps to be undertaken in order to avoid a recurrence of my illness.

Definetely NOT (1); rather not (2); I do not know (3); rather yes (4); definitely YES (5)

6. The thought about my illness scares me.

Definetely NOT (1); rather not (2); I do not know (3); rather yes (4); definitely YES (5)

7. I maintain positive thinking about the future.

Definetely NOT (1); rather not (2); I do not know (3); rather yes (4); definitely YES (5)

8. I feel as if my illness took everything away from me. Definetely NOT (1); rather not (2); I do not know (3); rather yes (4); definitely YES (5)

\section{Statistical methods}

The procedure of validation of the survey was carried out firstly on the basis of all 24 questions and then on questions belonging to each part separately. We assumed the significance of all statistical tests at $\alpha=0.05$. The Shapiro-Wilk test was used to assess the distribution of total score and the scores of each part of the survey. To check the internal consistency of the questionnaire the $\alpha$-Cronbach coefficient was applied. Factor analysis was performed using principal component analysis with Varimax rotation. The Catell criterion and Kaiser criterion were considered in order to determine components of the questionnaire. The factor analysis was preceded by checking: the determinant of correlation matrix, Kaiser-Mayer-Olkin (K-M-O) statistic and the Bartlett' test of sphericity. The scale and subscale levels were determined based on the percentiles scale.

\section{Results}

According to the Shapiro-Wilk test the distribution of total score and the scores of each part of the survey were statistically significant $(p<0.001)$ and we observed left-skewed distribution of the total score with kurtosis 0.29 and skewness of $-0,5$.

The value of the $\alpha$-Cronbach coefficient for all 24 questions was 0.855 indicating that the questionnaire is reliable and homogenous. Apart from that, we investigated $\alpha$-Cronbach coefficient value by removing individual questions. The coefficient's value was even higher when three questions (1,2 and 5) from part two were removed. We noted the value of $\alpha$-Cronbach $0.86,0.855,0.856$ respectively. As the $\alpha$-Cronbach coefficient's value in general was high for whole questionnaire, we decided not to remove any questions.

The next procedure which we implemented to validate the questionnaire was the factor analysis [14]. Generally, in case of occurrence of a high value of $\alpha$-Cronbach coefficient $(0.855)$ the factor analysis is not required, as the only one component is loaded by majority of items. Nonetheless, we made the factor analysis. The set of all 24 questions fulfilled the requirement of the factor analysis, i.e. value of the determinant of the correlation matrix was 0.001 , Kaiser-Mayer-Olkin (K-M-O) parameter was 0.843 and the Bartlett' test of sphericity was statistically significant. We carried out factor analysis by principal component analysis with Varimax rotation. We set the components of the questionnaire using the Catell criterion (based on the scree plot presented in the Fig. 1).

We could choice only one component [14-15]. The analysis of factor loadings showed that in the majority of items only one component is loaded.

\section{Interpretation of FCIS results}

The research conducted with the questionnaire assumed implementation of a scale to measure the strength or level of the investigated aspects. As it was mentioned above, there were scores from 1 to 5 assigned to each answer. The maximal score was then 120 points. For each part of the questionnaire, the total score for each subscale was 40 points. The score of the scale and scores of the subscales showed skewed distributions (figure 2) and they were statistically significant according to results of Shapiro-Wilk test $(p<0.001)$. Thanks to this, we determined the scale levels based on percentile scale. The percentiles determined for the scale and for the subscales are presented in Tab. 1.

Considering the scores related to the answers given by the surveyed persons, we assumed that the score below 30 percentile indicates low physical and mental functioning, the score between 30 and 70 percentile indicates medium physical and mental functioning and the score higher than 70 percentile indicates high physical and mental functioning. Alternatively, scoring less than 79 points for the entire questionnaire indicates 


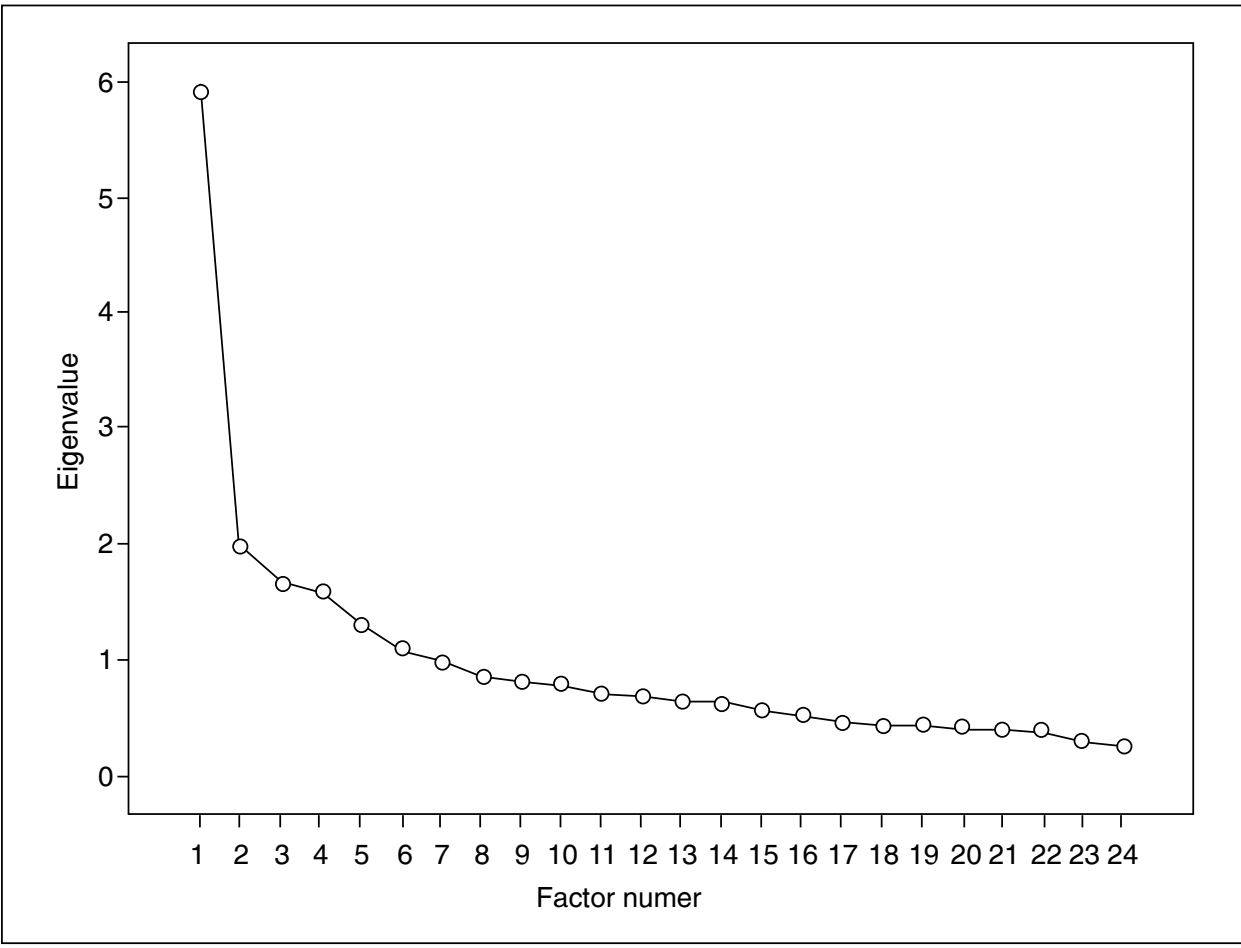

Figure 1. Scree plot

Table 1. The scale's levels of FCSI

Panel A

Score interpretation

(Part 1 + Part 2 + Part 3)

\begin{tabular}{ll}
\hline Score & Percentile levels \\
\hline$\leq 78$ & low level \\
$79-93$ & medium level \\
$\geq 94$ & high level
\end{tabular}

Maximal score - good functioning in the illness

Minimal score - bad functioning in the illness

\section{Panel B}

Score interpretation - Part 1: The impact of illness on the patient

\begin{tabular}{ll}
\hline Score & Percentile levels \\
\hline$\leq 23$ & low level \\
$24-33$ & medium level \\
$\geq 34$ & high level
\end{tabular}

Maximal score - illness does not affect the patient's functioning Minimal score - illness severely affects the patient's functioning

low functioning, scoring between 79 and 93 points indicates medium functioning and scores over 93 points are classified as high functioning. In case of the first

\section{Panel C}

Score interpretation - Part 2: The patient's impact on the illness.

\begin{tabular}{ll}
\hline Score & Percentile levels \\
\hline$\leq 24$ & low level \\
$25-29$ & medium level \\
$\geq 30$ & high level
\end{tabular}

Maximal score - the patient believes to have significant impact on the course of illness

Minimal score - the patient believes to have no impact on the course of illness

\section{Panel D}

Score interpretation - Part 3: The impact of illness on patient's attitude

\begin{tabular}{ll}
\hline Score & Percentile levels \\
\hline$\leq 27$ & low level \\
$28-33$ & medium level \\
$\geq 34$ & high level
\end{tabular}

Maximal score - the patient holds a very optimistic view for the future Minimal score - the patient holds a very pessimistic view for the future

subscale evaluating the impact of the disease on patient the scores under 23 points indicate the low level, scores between 24 and 33 are allocated to the medium 


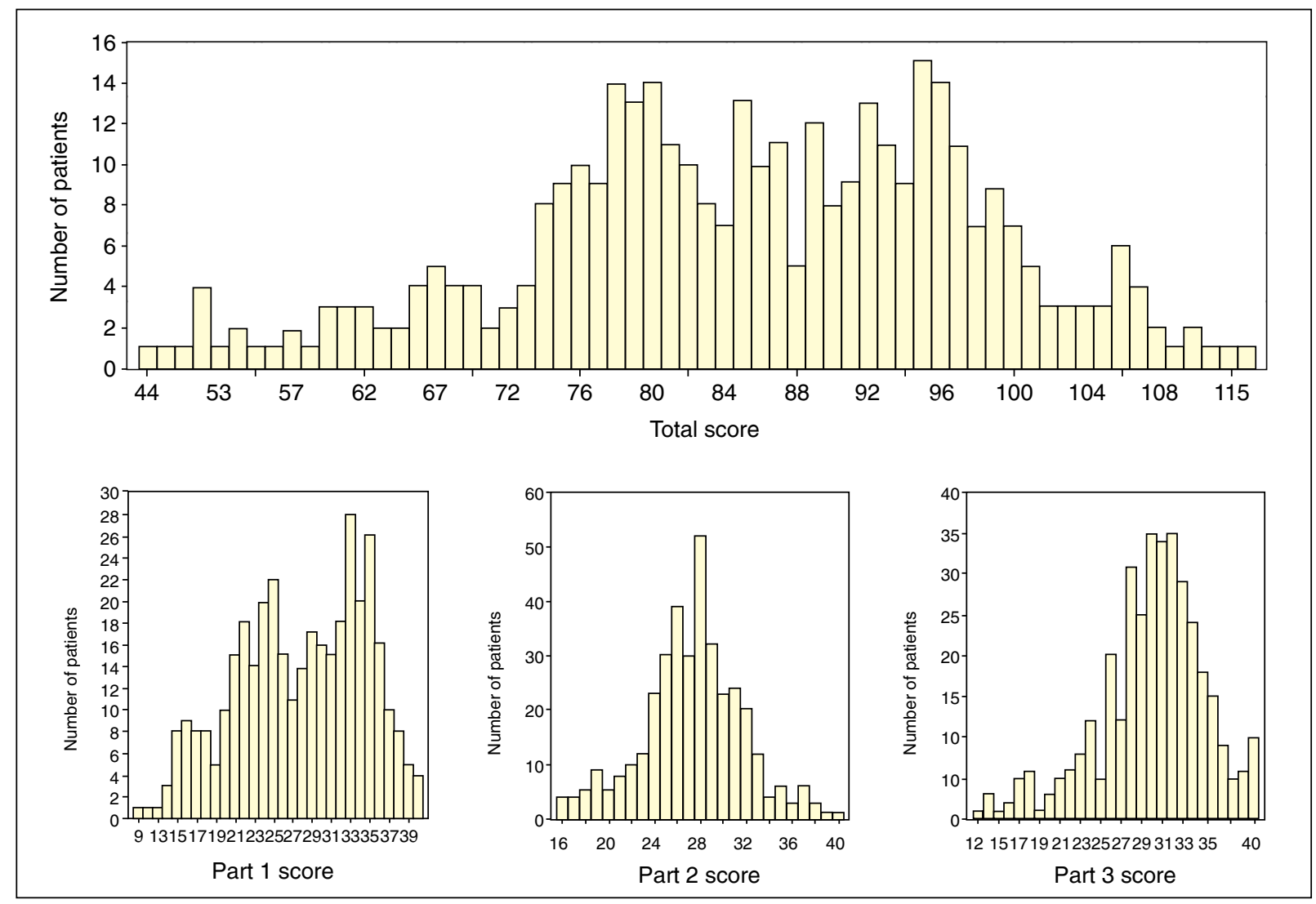

Figure 2. The distribution of the: total score of the FCIS, the impact of the disease on patient (Part 1), the patient's impact on the disease (Part 2), the disease on the patient's attitudes (Part 3)

level and scores over 34 points are classified as the high level (Tab. 1). The second subscale describing the patient's impact on the disease, the scores under 24 points indicate the low level, scores between 25 and 29 correspond to the medium level and the scores over 30 points indicate high level (Tab. 1). The impact of the disease on the patient's attitudes is evaluated in the third subscale. In this case 27 points indicate low level, scores between 28 and 33 points present medium level and scores over 33 points indicate high level. The distribution of total score and the scores of the subscales of FCIS are presented in Fig. 2.

\section{Discussion}

The authors of the study conducted a validation procedure for the Functioning in the Chronic Illness Scale (FCIS), a simple self-related questionnaire developed by A. Kubica. According to our knowledge FCIS is the first tool allowing the comprehensive assessment of physical and mental functioning dedicated for patients with chronic diseases.
The previously used tools (eg. WHO-DAS II scale) to assess physical and mental functioning were developed for people with disabilities. However, despite this, the WHO-DAS II scale was also applied in chronically ill patients with low back pain [16-17]. The CIA questionnaire is another tool for assessment of psychosocial functioning in patients with nutrition disorders [18-19]. Use of such tools for functioning evaluation of patients with chronic illness seems to be inappropriate, as originally these questionnaires were created to diagnose functioning in very specific situations.

The FCIS consisting of 24 items is characterized by a high level of reliability was shown to have a high reliability and the homogeneity (Cronbach's alpha coefficient $=0.855)$. The FCIS was divided into three subscales evaluating: the impact of the disease on the patient, the patient's impact on the disease and the impact of the disease on the patient's attitudes.

The first part of the questionnaire - the subscale evaluating the impact of the disease on patient is characterized by a high reliability (Cronbach's Alpha $=0.841$ ). It mainly refers to the patient's physical efficiency, quality of life and acceptance of the disease. These 
aspects of functioning of patients with chronic illness were evaluated in several studies [20-21]. Carson et al. [20] showed a relationship between the severity of heart failure symptoms according to the NYHA classification and the quality of life of patients assessed using the MLHFQ questionnaire, while Juenger et al. [21] applied the SF-36v2 questionnaire for the same purpose. The influence of chronic diseases on the disease acceptance was evaluated using the AIS questionnaire and on the quality of life using the WHOQoL questionnaire in subjects with respiratory diseases [22] and permanent atrial fibrillation [23] or using the SF-36v2 questionnaire in patients with diabetic neuropathy [24]. The first part of the FCIS evaluates also the functioning in terms of physical efficiency, which is currently widely investigated using the ADL scale in both patients [25] and healthy subjects [26]. To assess physical efficiency in people with chronic diseases, the EuroQol EQ-5D scale was proposed, which apart from the physical aspect evaluates the patient's well-being [27]. The ILQ questionnaire was used by Oris et al. [28] for the assessment of impact of chronic disease on patients' lifestyle including some physical and mental aspects.

The second and third subscales of the FCIS (characterized by Cronbach's alpha coefficients of 0.569 and 0.744 respectively) assessing the patient's impact on the disease and the impact of the disease on patient's attitudes refer mainly to the study of self-efficacy and the location of health control. The MHLC questionnaire, a commonly accepted tool for examining the location of health control, is often used together with questionnaires for the quality of life assessment in chronically ill patients. Sengul et al. [29] used the MHLC questionnaire with the WHOQoL questionnaire to examine patients with chronic low back pain, Wielenga - Boiten [30] in people with post-traumatic brain injury, and Basińska [13] in subjects with Hashimoto's disease. The GSES questionnaire is a tool for assessing one's self-effectiveness. In the study published by Cramm et al. [31], this questionnaire was used to assess young people with chronic illness. Maeda [32] used this tool in patients with heart failure together with evaluation of adherence to treatment. The PAM-13 questionnaire was also applied to measure the patient's activity in terms of self-efficacy and the location of health control [33].

This questionnaire is characterized by a high Cronbach's alpha in chronically ill patients: 0.870 in the Norwegian study by Moljord et al. [33] and 0.860 in the study carried out in Singapore by Ngooi et al. [34]. Rydlewska et al. [35] and Siennicka et al. [36] conducted researches in patients with chronic illness with simultaneous assessment of the location of health control and self-efficacy with separate tools.

Numerous studies indicate the need for the combined use of various tools for the overall assessment of various aspects of the functioning of subjects with chronic disease. Therefore, we assumed that there is a urgent need for one tool allowing for comprehensive and practically useful evaluation of such patients. The FICS is a response to this demand. It allows the examination of patients in various aspects of functioning with chronic disease in a quick and simple way, without use of several different tools. Such an approach should allow to diagnose deficit areas in patients and to implement appropriate interventions by the members of the therapeutic team. The functioning of patients with chronic disease is closely related to medication adherence and has a strong impact on clinical outcome [37-42] .

\section{Conclusion}

The validation procedure revealed that $\mathrm{FCIS}$ is a reliable and homogeneous tool to measure patient's physical and mental functioning in the chronic illness. The set of items divided into 3 subscales allows evaluation of: the impact of the disease on the patient, the patient's impact on the disease and the impact of the disease on the patient's attitudes.

\section{References}

1. 1.Olano-Lizarraga M, Oroviogoicoechea C, Errasti-Ibarrondo B, et al The personal experience of living with chronic heart failure: a qualitative meta-synthesis of the literature. J Clin Nurs. 2016; 25(17-18): 2413-2429, doi: 10.1111/jocn.13285, indexed in Pubmed: 27273246.

2. Hobbs FDR, Kenkre JE, Roalfe AK, et al. Impact of heart failure and left ventricular systolic dysfunction on quality of life: a cross-sectional study comparing common chronic cardiac and medical disorders and a representative adult population. Eur Heart J. 2002; 23(23): 1867-1876, indexed in Pubmed: 12445536.

3. Rogan A, McCarthy K, McGregor G, et al. Quality of life measures predict cardiovascular health and physical performance in chronic renal failure patients. PLoS One. 2017; 12(9): e0183926, doi: 10.1371/journal.pone.0183926, indexed in Pubmed: 28910330.

4. Pagels AA, Söderkvist BK, Medin C, et al. Health-related quality of life in different stages of chronic kidney disease and at initiation of dialysis treatment. Health Qual Life Outcomes. 2012; 10: 71, doi: 10.1186/14777525-10-71, indexed in Pubmed: 22710013.

5. Oris L, Luyckx K, Rassart J, et al. Illness Identity in Adults with a Chronic Illness. J Clin Psychol Med Settings. 2018 [Epub ahead of print], doi: 10.1007/s10880-018-9552-0, indexed in Pubmed: 29468569

6. Novak Sarotar B, Lainscak M. Psychocardiology in the elderly. Wien Klin Wochenschr. 2016; 128(Suppl 7): 474-479, doi: 10.1007/s00508016-1139-x, indexed in Pubmed: 27896465.

7. O'Neil A, Stevenson CE, Williams ED, et al. The health-related quality of life burden of co-morbid cardiovascular disease and major depressive disorder in Australia: findings from a population-based, cross-sectional study. Qual Life Res. 2013; 22(1): 37-44, doi: 10.1007/s11136-0120128-4, indexed in Pubmed: 22323040.

8. Kunschitz E, Friedrich O, Schöppl C, et al. Assessment of the need for psychosomatic care in patients with suspected cardiac disease. Wien Klin Wochenschr. 2017; 129(7-8): 225-232, doi: 10.1007/s00508-0161050-5, indexed in Pubmed: 27495803.

9. Romera I, Perez V, Menchón JM, et al. Social and occupational functioning impairment in patients in partial versus complete remission of a major depressive disorder episode. A six-month prospective epidemiological study. Eur Psychiatry. 2010; 25(1): 58-65, doi: 10.1016/j. eurpsy.2009.02.007, indexed in Pubmed: 19553092.

10. Burström M, Brännström M, Boman $K$, et al. Life experiences of security and insecurity among women with chronic heart 
failure. J Adv Nurs. 2012; 68(4): 816-825, doi: 10.1111/j.1365-2648.2011.05782.x, indexed in Pubmed: 21733141.

11. Alla F, Briançon S, Guillemin F, et al. EPICAL Investigators. Self-rating of quality of life provides additional prognostic information in heart failure. Insights into the EPICAL study. Eur J Heart Fail. 2002; 4(3): 337-343, indexed in Pubmed: 12034160

12. Pedrosa $\mathrm{H}, \mathrm{Sa} A D e$, Guerreiro M, et al. Functional evaluation distinguishes $\mathrm{MCl}$ patients from healthy elderly people - The ADCS/MCI/ADL scale. The journal of nutrition, health \& aging. 2010; 14(8): 703-709, doi: 10.1007/s12603-010-0102-1.

13. Basinska MA, Andruszkiewicz A. Health locus of control in patients with graves-basedow disease and hashimoto disease and their acceptance of illness. Int J Endocrinol Metab. 2012; 10(3): 537-542, doi 10.5812/ijem.3932, indexed in Pubmed: 23843816.

14. Castello AB, Osborne JW. Best Practices in Exploratory Factor Analysis Four Recommendations for Getting the Most from Your Analysis. Practical Assessment, Research \& Evaluation. 2005; 10(7)

15. Rowe P. Essential Statistics for the Pharmaceutical Sciences. 2015 doi: 10.1002/9781119109075

16. Roe C. Retaining the patient perspective in the International Classification of Functioning. Disability and Health Core Set for low back pain. Patient Preference and Adherence. 2008: 337, doi: 10.2147/ppa.s4419.

17. Garin O, Ayuso-Mateos JL, Almansa J, et al. MHADIE consortium Validation of the ,World Health Organization Disability Assessment Schedule, WHODAS-2" in patients with chronic diseases. Health Qual Life Outcomes. 2010; 8: 51, doi: 10.1186/1477-7525-8-51, indexed in Pubmed: 20482853.

18. Bohn K, Doll HA, Cooper Z, et al. The measurement of impairment due to eating disorder psychopathology. Behav Res Ther. 2008; 46(10): 1105-1110, doi: 10.1016/j.brat.2008.06.012, indexed in Pubmed: 18710699

19. Bohn K. Clinical Impairment Assessment Questionnaire (CIA). Encyclopedia of Feeding and Eating Disorders. 2017: 126-129, doi: 10.1007/978-981-287-104-6 85.

20. Carson P, Tam SW, Ghali JK, et al. Relationship of quality of life scores with baseline characteristics and outcomes in the African-American heart failure trial. J Card Fail. 2009; 15(10): 835-842, doi: 10.1016/j. cardfail.2009.05.016, indexed in Pubmed: 19944359

21. Juenger J. Health related quality of life in patients with congestive hear failure: comparison with other chronic diseases and relation to functional variables. Heart. 2002; 87(3): 235-241, doi: 10.1136/heart.87.3.235.

22. Kurpas D, Mroczek B, Knap-Czechowska H, et al. Quality of life and acceptance of illness among patients with chronic respiratory diseases. Respir Physiol Neurobiol. 2013; 187(1): 114-117, doi: 10.1016/j. resp.2013.02.009, indexed in Pubmed: 23419519

23. Jankowska-Polańska B, Kaczan A, Lomper K, et al. Symptoms, acceptance of illness and health-related quality of life in patients with atrial fibrillation. Eur J Cardiovasc Nurs. 2018; 17(3): 262-272, doi: 10.1177/1474515117733731, indexed in Pubmed: 28933196.

24. Lewko J, Polityńska B, Kochanowicz J, et al. Quality of life and its relationship to the degree of illness acceptance in patients with diabetes and peripheral diabetic neuropathy. Adv Med Sci. 2007; 52 Suppl 1 : 144-146, indexed in Pubmed: 18229653.

25. Benetos A, Gautier S, Labat C, et al. Mortality and cardiovascular events are best predicted by low central/peripheral pulse pressure amplification but not by high blood pressure levels in elderly nursing home subjects: the PARTAGE (Predictive Values of Blood Pressure and Arterial Stiffness in Institutionalized Very Aged Population) study. J Am Coll Cardiol. 2012; 60(16): 1503-1511, doi: 10.1016/j.jacc.2012.04.055, indexed in Pubmed: 22999729.

26. Pedrosa H, Sa ADe, Guerreiro M, et al. Functional evaluation distinguishes $\mathrm{MCl}$ patients from healthy elderly people - The ADCS/MCI/ADL scale. The journal of nutrition, health \& aging. 2010; 14(8): 703-709, doi: 10.1007/s12603-010-0102-1.
27. Devlin NJ, Brooks R. EQ-5D and the EuroQol Group: Past, Present and Future. Appl Health Econ Health Policy. 2017; 15(2): 127-137, doi: 10.1007/s40258-017-0310-5, indexed in Pubmed: 28194657.

28. Oris L, Luyckx K, Rassart J, et al. Illness Identity in Adults with a Chronic Illness. J Clin Psychol Med Settings. 2018 [Epub ahead of print], doi: 10.1007/s10880-018-9552-0, indexed in Pubmed: 29468569.

29. Sengul Y, Kara B, Arda MN. The relationship between health locus of control and quality of life in patients with chronic low back pain. Turk Neurosurg. 2010; 20(2): 180-185, doi: 10.5137/1019-5149.JTN.261609.1, indexed in Pubmed: 20401846.

30. Wielenga-Boiten JE, Heijenbrok-Kal MH, Ribbers GM. The Relationship of Health Locus of Control and Health-Related Quality of Life in the Chronic Phase After Traumatic Brain Injury. J Head Trauma Rehabil. 2015; 30(6): 424-431, doi: 10.1097/HTR.0000000000000128, indexed in Pubmed: 25699627

31. Cramm JM, Strating MMH, Roebroeck ME, et al. The Importance of General Self-Efficacy for the Quality of Life of Adolescents with Chronic Conditions. Soc Indic Res. 2013; 113(1): 551-561, doi: 10.1007/s11205-012-0110-0, indexed in Pubmed: 23874059.

32. Maeda U, Shen BJ, Schwarz ER, et al. Self-efficacy mediates the associations of social support and depression with treatment adherence in heart failure patients. Int J Behav Med. 2013; 20(1): 88-96, doi: 10.1007/s12529-011-9215-0, indexed in Pubmed: 22212607.

33. Moljord IE, Lara-Cabrera ML, Perestelo-Pérez L, et al. Psychometric properties of the Patient Activation Measure-13 among out-patients waiting for mental health treatment: A validation study in Norway. Patient Educ Couns. 2015; 98(11): 1410-1417, doi: 10.1016/j. pec.2015.06.009, indexed in Pubmed: 26146239.

34. Ngooi BiX, Packer TL, Kephart G, et al. Validation of the Patient Activation Measure (PAM-13) among adults with cardiac conditions in Singapore. Qual Life Res. 2017; 26(4): 1071-1080, doi: 10.1007/s11136016-1412-5, indexed in Pubmed: 27645458

35. Rydlewska A, Krzysztofik J, Libergal J, et al. Health locus of control and the sense of self-efficacy in patients with systolic heart failure: a pilot study. Patient Prefer Adherence. 2013; 7: 337-343, doi: 10.2147/PPA. S41863, indexed in Pubmed: 23723691.

36. Siennicka AE Gościńska-Bis K, Wilczek J et al. Perception of health control and self-efficacy in heart failure. Kardiol Pol. 2016; 74(2): 168-178, doi: 10.5603/KP.a2015.0137, indexed in Pubmed: 26202531

37. Kubica A Obońska K, Fabiszak T, et al. Adherence to antiplatelet treatment with $\mathrm{P} 2 \mathrm{Y} 12$ receptor inhibitors. Is there anything we can do to improve it? A systematic review of randomized trials. Curr Med Res Opin. 2016; 32(8): 1441-1451, doi: 10.1080/03007995.2016.1182901, indexed in Pubmed: 27112628.

38. Kubica A, Kasprzak M, Obońska K, et al. Discrepancies in assessment of adherence to antiplatelet treatment after myocardial infarction. Pharmacology. 2015; 95(1-2): 50-58, doi: 10.1159/000371392, indexed in Pubmed: 25592409

39. Kubica A, Kasprzak M, Siller-Matula J, et al. Time-related changes in determinants of antiplatelet effect of clopidogrel in patients after myocardial infarction. Eur J Pharmacol. 2014; 742: 47-54, doi: 10.1016/j. ejphar.2014.08.009, indexed in Pubmed: 25199965.

40. Kosobucka A, Michalski P, Pietrzykowski $k$ et al. Adherence to treatment assessed with the Adherence in Chronic Diseases Scale in patients after myocardial infarction. Patient Prefer Adherence. 2018; 12: 333-340, doi: 10.2147/PPA.S150435, indexed in Pubmed: 29551891

41. Kubica A, Kosobucka A, Michalski P, et al. TheAdherenceinChronic Diseases Scale - a new tool to monitor implementation of a treatment plan. Folia Cardiol. 2017; 12: 19-26, doi: 10.5603/FC.2016.0000

42. Kubica A, Obońska K, Kasprzak M, et al. Prediction of high risk of non-adherence to antiplatelet treatment. Kardiol Pol. 2016; 74(1) 61-67, doi: 10.5603/KP.a2015.0117, indexed in Pubmed: 26101025. 